\title{
Fibromatosis gingival hereditaria: una rara enfermedad. Reporte de una familia
}

Hereditary Gingival Fibromatosis:

A Rare Disease. A Family Report

Fibromatose gengival hereditária uma estranha doença: reporte de uma família

\author{
Edwin Guzmán Rivera, OD"; \\ Ary López Álvarez, OD²; \\ Jonathan Harris Ricardo OD, MSc ${ }^{* 1,2}$
}

Recibido: 26 de julio de 2017 / Aceptado: 26 de febrero de 2018

Doi: http://dx.doi.org/10.12804/revistas.urosario.edu.co/revsalud/a.6775

Para citar este artículo: Guzmán Rivera E, López Álvarez A, Harris Ricardo J. Fibromatosis gingival hereditaria: una rara enfermedad. Reporte de una familia. Rev Cienc Salud. 2018;16(2):368-375. Doi: http://dx.doi.org/10.12804/revistas.urosario.edu.co/revsalud/a.6775

\section{Resumen}

Introducción: la fibromatosis gingival hereditaria es un desorden genético raro que produce un sobrecrecimiento gingival y el desplazamiento dental asociado, la patogénesis y la base molecular de la enfermedad sigue siendo desconocida. Dado que es una enfermedad poco frecuente, es importante que el profesional en el área de la salud oral conozca las características clínicas, histológicas y genéticas de la enfermedad con el objetivo de realizar un correcto diagnóstico, plan de tratamiento y orientación sobre la condición de la patología. Presentación del caso: se reporta informe de una familia con tres generaciones afectadas con fibromatosis gingival hereditaria, en la que se describen las características clínicas, histopatológicas y tratamiento. Conclusión: la fibromatosis gingival hereditaria es un trastorno poco frecuente que genera diversos grados de aumento en el volumen gingival, los compromisos estéticos y funcionales a menudo requieren intervención quirúrgica, histológicamente es común la presencia de abundantes haces de colágeno y fibroblastos.

Palabras clave: fibromatosis gingival, familia, genética, cirugía bucal.

\section{Abstract}

Introduction: Hereditary gingival fibromatosis is a rare genetic disorder that produces a gingival overgrowth and the associated dental displacement, the pathogenesis and the molecular basis of the

\footnotetext{
1 Universidad de Cartagena.

2 Corporación Universitaria Rafael Núñez.

* Autor de correspondencia: j.harris.r@hotmail.com
} 
disease remains unknown. Given that it is a rare disease, it is important that the professionals in the field of oral health know the clinical, histological and genetic characteristics of the disease in order to make a correct diagnosis, a treatment plan and the guidance on the condition of the pathology. Case presentation: Report of a family with three generations affected with hereditary gingival fibromatosis, in which the clinical, histopathological and treatment characteristics are described. Conclusions: Hereditary gingival fibromatosis is a rare disorder that generates varying degrees of increase in gingival volume, aesthetic and functional compromises often require surgical intervention, the presence of abundant bundles of collagen and fibroblasts is histologically common.

Keywords: fibromatosis, gingival, family, genetics, oral surgery.

\section{Resumo}

Introdução: a fibromatose gengival hereditária é uma desordem genética estranha que produz um sobrecrescimento gengival e o deslocamento dental associado, a patogênese e a base molecular da doença segue sendo desconhecida. Dado que é uma doença pouco frequente, é importante que o profissional na área da saúde oral conheça as características clínicas, histológicas e genéticas da doença com o objetivo de realizar um correto diagnóstico, plano de tratamento e orientação sobre a condição da patologia. Apresentação do caso: reporta-se o informe de uma família com três gerações afetados com fibromatose gengival hereditária, no que se descrevem as características clínicas, histopatológicas e tratamento. Conclusão: a fibromatose gengival hereditária é um transtorno pouco frequente que gera diversos graus de aumento no volume gengival, os compromissos estéticos e funcionais frequentemente requerem intervenção cirúrgica, histologicamente é comum a presença de abundantes feixes de colágeno e fibroblastos.

Palavras-chave: fibromatose gengival, família, genética, cirurgia bucal.

\section{Introducción}

工 a fibromatosis gingival hereditaria ( $\mathrm{FGH}$ es una patología que afecta de manera directa las tiva, de carácter benigno, que presenta una incidencia de 1 en 750000 (1).

La FGH se presenta de forma aislada, no se encuentra asociada con síndromes, fármacos o placa dental, la presencia de antecedentes familiares con la enfermedad es importante para su impresión clínica, se le relaciona con síndromes como Cowden, Zimmerman-Laband y Murray-Puretic (2). Con los estudios moleculares se descubrieron cambios celulares asociados con la FGH, pese a esto, la patogénesis de la enfermedad es todavía desconocida, a la mutación de gen sos1 se le ha relacionado con una forma de la enfermedad; sin embargo, la FGH muestra heterogeneidad genética, ya que se han determinado mutaciones en varios genes, en los estudios de ligamiento se han localizado lugares de aislamiento en el cromosoma 2p21-p22 y el 5q13-q22 (3).

La patología se caracteriza por afectar las encías, lo cual causa un aumento excesivo de tamaño del tejido gingival y se presenta de forma generalizada, de aspecto fibroso, consistencia firme, no sangrante y asintomática, no tiene predilección por genero sexual $(1,4)$. El exagerado 
crecimiento gingival origina problemas funcionales y estéticos, como desplazamiento dental, diastemas, dificultad en el acto masticatorio, retraso en la erupción dentaria, entre otros (5).

Histológicamente, presenta abundantes fibras colágenas y fibroblastos, Kather et al. reportaron focos de hueso y pequeñas partículas calcificadas en la muestra patológica (6). La terapéutica quirúrgica es la más empleada mediante la gingivectomía y gingivoplastía, con ella se disminuye el grosor y altura de los tejidos gingivales, por lo que presenta una alta frecuencia de recurrencia (7).

El propósito del trabajo es concientizar a los profesionales en el área de la salud de la existencia de una enfermedad huérfana, para así conocer parámetros clínicos, histopatológicos, exámenes de laboratorio y tratamiento.

\section{Presentación del caso}

$\Gamma^{l}$ estudio de la familia se realizó a partir de un paciente de sexo femenino de 13 años de Eedad, de la segunda generación, la cual acudió a la Clínica Odontológica de la Corporación Universitaria Rafael Núñez por presentar incremento generalizado en el tamaño del tejido gingival, lo que afectaba el maxilar y la mandíbula. En el interrogatorio, la madre y un hermano reportaron antecedentes de la misma condición, los miembros de la familia que participaron en estudio dieron la aprobación al firmar el consentimiento informado.

La estructura del árbol genealógico mostró que la madre presentó manifestaciones de la enfermedad; de la primera generación, un sujeto masculino y otro femenino presentaron características clínicas de la patología; mientras que en la segunda generación solo se presentó un afectado de sexo masculino (figura 1).

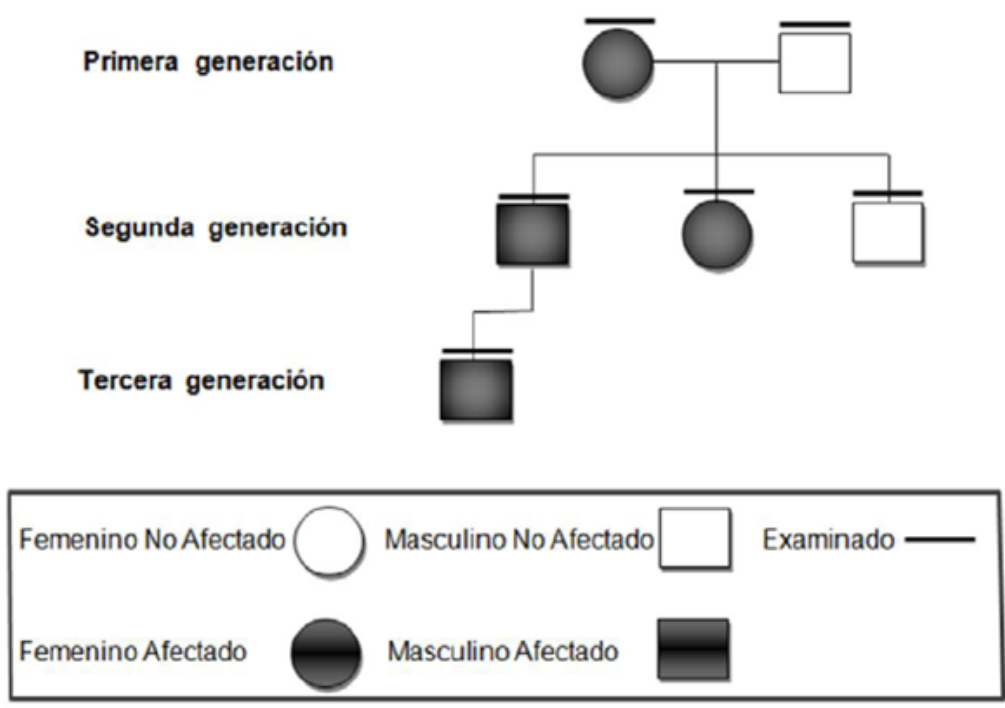

Figura 1. Árbol genealógico de FGH 
Todos los individuos fueron evaluados, se les realizó examen intrabucal en el que se tuvo en cuenta, para ser calificado como afectado, aumento en el volumen de la encía que cubriera al menos un tercio de la corona dental, en mínimo de cinco dientes, que no estuvieran consumiendo medicamentos relacionados con el crecimiento gingival - como la fenitoína, los bloqueadores de los canales de calcio y la ciclosporina A-, que al ser evaluados por medicina especializada no presentaran alteraciones físicas, sistémicas, renales, hepáticas, ni relación con síndromes o que manifestaran antecedentes de la FGH.

\section{Presentación de afectado 1}

En la inspección intrabucal, la madre presentó aumento en el tamaño del tejido gingival de la zona posterosuperior e inferior, lo que afectaba la encía papilar, marginal y adherida, de color rosa pálido, superficie lisa, cubriendo un tercio de la corona dental, de consistencia firme, asintomática a la palpación, ausencia de sangrado y de once meses de evolución, se observó biofilm supragingival en los molares del maxilar (figura 2), radiográficamente se observó destrucción coronal completa y ensanchamiento del ligamento periodontal de los dientes 12, 23, 26 y 27 , desplazamiento dental de los dientes 31, 32, 41 y 42.

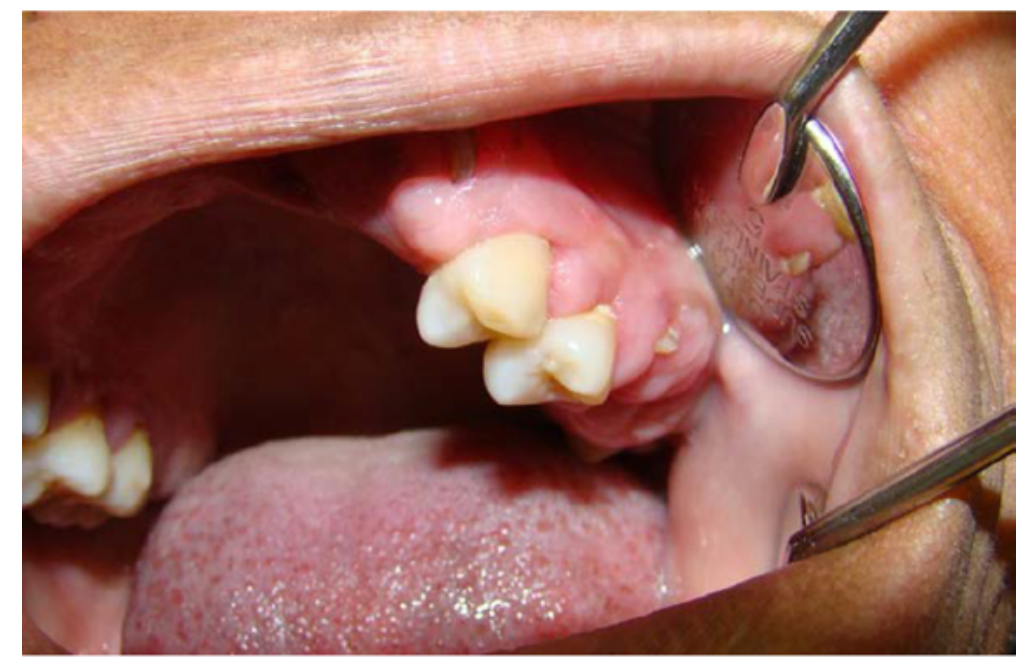

Figura 2. Aumento en el tamaño en el tejido gingival

\section{Presentación de afectado 2}

En la exploración que se realizó en el sujeto de sexo masculino de la primera generación, se notó aumento en el volumen de la encía en el sector anteroinferior, que cubre dos tercios de la corona de los dientes del mismo color de la mucosa adyacente, consistencia firme, superficie irregular, desplazamiento de las estructuras dentarias. Se observó biofilm supragingival generalizado en los dientes de la mandíbula y maxilar (figura 3). El estudio radiográfico mostró cambios específicos como desplazamiento de los dientes 31, 32, 33, 41, 42 y 43 y reabsorción radicular del diente número 41. 


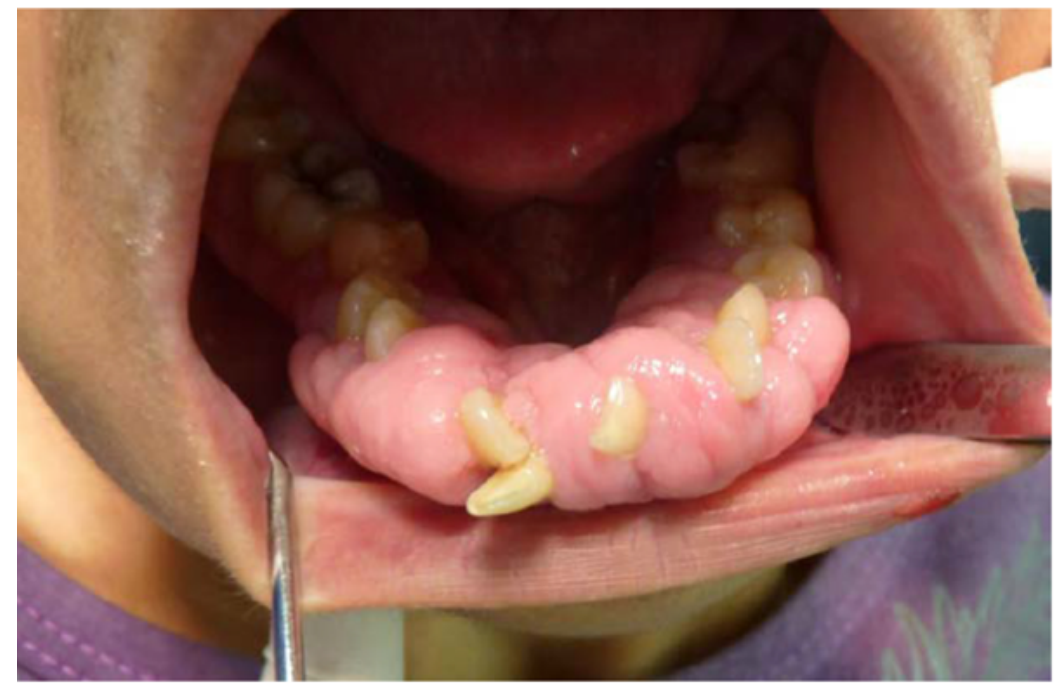

Figura 3. Aumento en el volumen de la encía en el sector anteroinferior

\section{Presentación de afectado 3}

En la paciente femenina de la primera generación, se observó agrandamiento gingival generalizado que cubría el $80 \%$ de la corona dental, de superficie irregular, del mismo color de la mucosa adyacente de consistencia firme, apariencia fibromatosa, no sangrante, de aproximadamente un año de evolución, asintomático, le originaba discapacidad funcional y estética, se observó biofilm supragingival en los molares del maxilar y mandíbula (figura 4). En la radiografía panorámica se observaron dientes en buen estado general y la presencia del diente número 73.

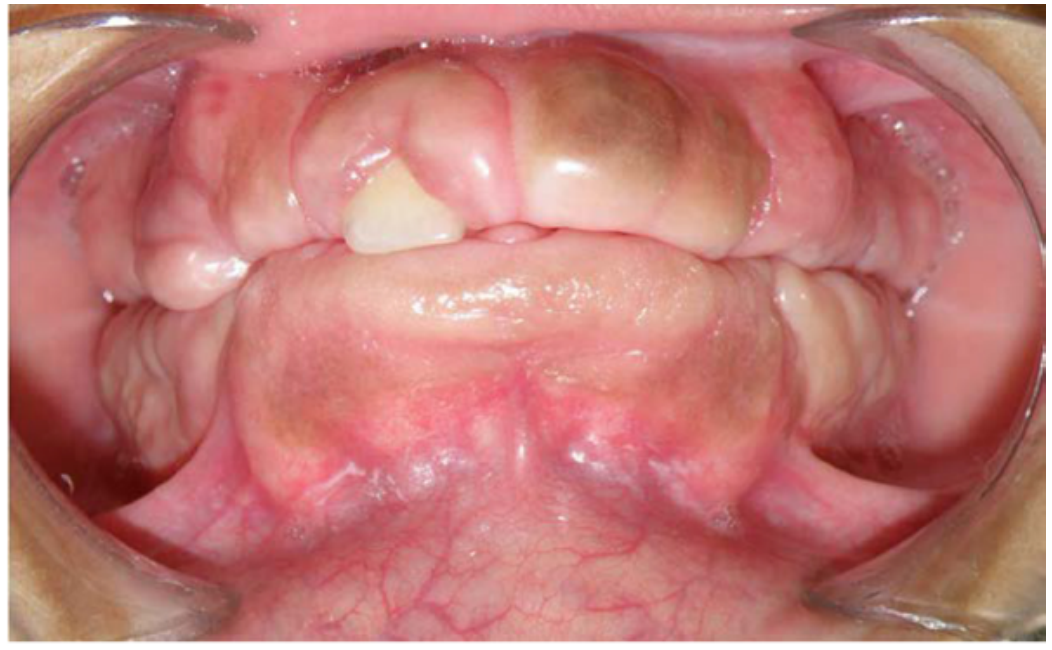

Figura 4. Agrandamiento gingival generalizado

Nota: se encuentra cubriendo la corona de los dientes, de superficie irregular, del mismo color de la mucosa adyacente y consistencia firme. 


\section{Presentación de afectado 4}

Al momento de realizar el examen clínico al integrante de la tercera generación, no presentó aumento del tamaño de las encías, pero en la anamnesis el padre comentó antecedentes de la condición patológica y que recibió tratamiento quirúrgico para ello.

Todos los miembros de la familia se remitieron a medicina especializada para valoración, el cual reportó que se encuentran con buen desarrollo psicomotor, describe que en el examen físico no presentaron alteraciones, ni asociación con algún síndrome. Se ordenaron exámenes de laboratorio como hemograma completo, extendido de sangre periférica, creatinina, nitrógeno ureico y transaminasas como el got y gpt, los cuales se encontraron entre los valores normales, para descartar alteraciones sistémicas, renales y hepáticas.

Se estableció diagnóstico de fibromatosis gingival hereditaria, teniendo en cuenta los parámetros clínicos bucales, evaluación médica, exámenes de laboratorio y antecedentes familiares.

Se realizó tratamiento quirúrgico en los pacientes afectados, la técnica quirúrgica consistió en una gingivectomía, gingivoplastia y colocación de un tutor en acetato diseñado prequirúrgicamente en modelos de estudios con cemento quirúrgico periodontal, para proteger en forma física la superficie de la herida del trauma mecánico durante la cicatrización, evitar la formación de tejido excesivo de granulación y empaquetamiento de alimentos.

Las tres muestras de tejido fueron rotuladas en frascos con formol al $10 \%$ y enviadas al laboratorio patológico, el reporte microscópico describe fragmentos de mucosa oral revestidos por epitelio paraqueratósico, hiperplásico, sobre estroma con abundantes haces gruesos de colágeno dispuestos al azar, compatible con fibromatosis gingival, se espera confirmar la mutación del gen con pruebas moleculares

Se realizaron controles postquirúrgicos a los 7, 14 y 21 días, en los que se evidenció disminución en el volumen del tejido gingival, buen proceso de cicatrización, los pacientes manifestaron mejoras en el proceso de masticación.

\section{Discusión}

Ce ha informado como la causa de fGH una mutación de marco de lectura heterocigoto en Sos1, en general se transmite como un rasgo autosómico dominante, hasta la fecha cuatro loci 2p22.1, 5q13-q22, 2p23.3-p22.3 y 11p15 han sido asociados, siendo importante para el diagnóstico realizar principalmente un examen clínico, periodontal, un historial médico y familiar y pruebas de laboratorio (8).

Vishnoi et al. estudiaron una familia con antecedentes de FGH, revisaron cuatro generaciones con 33 sujetos, en donde se mostró claramente la forma autosómica dominante, ya que se encontraron afectados en la segunda generación ocho personas, en la tercera nueve y en la cuarta un sujeto, para un total de 16 afectados (9). Ese estudio coincide con el presente reporte, 
en el que en una familia con un total de seis miembros con antecedentes de FGH, se hallaron tres afectados (madre, hermano y hermana) y antecedentes del crecimiento gingival en el miembro de la segunda generación, lo que demuestra que es una patología heredada de condición autosómica dominante.

Akhilanand afirma que la FGH es una rara condición de los tejidos gingivales generalizados, caracterizado por el aumento en el volumen de la encía, que puede llegar a cubrir la totalidad de la corona dental y generar problemas estéticos y funcionales, se presenta de forma aislada no asociada con síndromes, infecciones, tratamientos farmacológico (10). Estas características clínicas son similares a las encontradas en tres casos del presente trabajo, ya que en varios pacientes se observó agrandamiento gingival generalizado, lo cual genero inconformidad estética al momento de sonreír o hablar, y funcional como lo son incompetencia labial, problemas al momento de realizar el acto masticatorio, retraso en la erupción de los dientes permanentes y dentición temporal tardía.

Bansal et al. resaltan la importancia de historia familiar en el diagnóstico de la FGH, lo que concuerda con el actual reporte basado en los datos aportados en el interrogatorio realizado en la historia clínica como la ingesta de medicamentos, la ausencia de procesos infecciosos, síndromes y la afección de la encía en diferentes integrantes de la familia (11).

Como alternativa de tratamiento, Dhadsey et al. realizaron una gingivectomía a bisel externo, en la que eliminaron el tejido gingival excesivo, y una gingivoplastia para devolverle la anatomía a este tejido y, luego, complementarlo con un efectivo programa de higiene oral (12). Esto coincidió con el tratamiento del presente trabajo, en el que se realizó gingivectomía y gingivoplastia para devolver la apariencia normal de la encía y luego controles postoperatorios en donde se evaluó la cicatrización y la salud oral del paciente.

\section{Conflicto de interés}

os autores declaran no tener conflicto de intereses; el trabajo fue financiado por los autores.

\section{Referencias}

1. Avelar RL, de Luna Campos GJ, de Carvalho Bezerra Falcão PG, da Costa Araújo FA, de Carvalho RW, de Souza Andrade ES. Hereditary gingival fibromatosis: a report of four cases in the same family. Quintessence Int. 2010;41(2):99-102.

2. Pego SP, Coletta RD, Mendes DC, de Faria PR, Melo-Filho MR, Alves LR, et al. Hereditary gingival fibromatosis: clinical and ultrastructural features of a new family. Med Oral Patol Oral Cir Bucal. 2015;20(2):150-5. 
3. Gawron K, Łazarz-Bartyzel K, Potempa J3, Chomyszyn-Gajewska M. Gingival fibromatosis: clinical, molecular and therapeutic issues. Orphanet J Rare Dis. 2016;27(11):9. doi: 10.1186/s13023-016-0395-1

4. Almiñana-Pastor PJ, Buitrago-Vera PJ, Alpiste-Illueca FM, Catalá-Pizarro M. Hereditary gingival fibromatosis: Characteristics and treatment approach. J Clin Exp Dent. 2017;9(4):e599-602. doi: 10.4317/jced.53644

5. Tripathi AK, Upadhaya V, Kumar V, Saimbi CS. Hereditary gingival fibromatosis and its management: 2-year follow-up. Contemp Clin Dent. 2014;5(4):569-71. doi: 10.4103/0976237X.142835

6. Kather J, Salgado MA, Salgado UF, Cortelli JR, Pallos D. Clinical and histomorphometric characteristics of three different families with hereditary gingival fibromatosis. Oral Surg Oral Med Oral Pathol Oral Radiol Endod. 2008;105(3):348-52.

7. Kanagotagi S, Sidana S, Rajguru S, Padhye A. Gingival Fibromatosis with Distinctive Facies - A Three Generation Case Report. J Clin Diagn Res. 2015;9(5):5-7. doi: 10.7860/ JCDR/2015/11670.5881

8. Gawron K, Łazarz-Bartyzel K, Potempa J, Chomyszyn-Gajewska M. Gingival fibromatosis: clinical, molecular and therapeutic issues. Orphanet J Rare Dis. 2016;11: 9. doi: 10.1186/s13023-016-0395-1

9. Vishnoi SL. Hereditary gingival fibromatosis: Report of four generation pedigree. International Journal of Case Reports and Images 2011;2(6):1-5. doi: 10.5348/ijcri-201106-36-CS-1

10. Chaurasia A. Hereditary gingival fibromatosis, Natl J Maxillofac Surg. 2014;5(1):42-6. doi: 10.4103/0975-5950.140171

11. Bansal A, Narang S, Sowmya K, Sehgal N. Treatment and two-year follow-up of a patient with hereditary gingival fibromatosis. J Indian Soc Periodontol. 2011;15(4):406-9. doi: 10.4103/0972-124X.92581

12. Dhadse P, Yeltiwar R, Pandilwar P, Gosavi S. Hereditary Gingival Fibromatosis. J Indian Soc Periodontol. 2012;16(4):606-9. doi: 10.4103/0972-124X.106930 